\title{
Biometrical and chromosome analyses of lines of Drosophila melanogaster selected for central excitation
}

\author{
Mark Vargo* and Jerry Hirsch $\dagger$
}

\author{
* Department of Genetics and Development, and \\ $\dagger$ Department of Psychology, and † Department of \\ Ecology, Ethology, and Evolution, University of \\ Illinois, Urbana-Champaign, IL 61820.
}

Lines of Drosophila melanogaster, bidirectionally selected for extreme and opposite expression of central excitatory state (CES), were subjected first to biometrical and then to chromosome analysis. The analyses revealed that (a) low CES expression is partially dominant to high, (b) at least two chromosomes (II and III) are correlated with CES expression, (c) for the low line, both chromosomes, II and III, are necessary for low expression, (d) cytoplasmic factors are involved with CES expression, and (e) loci of minor effect on the $X$ and $Y$ chromosomes are correlated with CES expression.

\section{INTRODUCTION}

Biometrical analysis has been used extensively in behaviour genetic studies to assess and quantify the genetic components of variance correlated with behavioural phenotypes. The result of these studies has been the estimation of additive, dominance, and epistatic components of variance correlated with the behaviour-trait under study. Though these estimates may prove useful in assessing the genetic architecture of the trait in a specified population of animals, they provide little information about the loci functioning to produce the behavioural expression.

Chromosome analysis can determine the effect each chromosome has on the phenotype through the isolation of homologous chromosome pairs from an inbred or selected line on an unselected genetic background (Robertson, 1954; Hirsch, 1959; Hirsch and Erlenmeyer-Kimling, 1962; Hirsch and Ksander, 1969; Pyle, 1978). The experimental groups produced in such an analysis can contain either single chromosome pairs or combinations of chromosome pairs. For example in Drosophila melanogaster, which has three major chromosomes, six experimental groups can be pro-

\footnotetext{
* Present address: Department of Biology, Brandeis University, Waltham, MA 02254.

The primary data used in this article are available in the first author's doctoral dissertation (Vargo, 1984).
}

duced; three with each chromosome isolated separately on a control background and three with each chromosome in combination (i.e., $\mathrm{X}$ and II, $X$ and III, and II and III).

Some studies have employed biometrical analysis in conjunction with other analyses to identify the genetic correlates of the phenotype (Mather and Harrison, 1949). What is described below are the results of a biometrical and chromosome analysis with central excitatory state (CES) as the behavior of interest. Excitatory state, for our purposes, is manifested in a food-deprived, watersatiated fly by an increased frequency of proboscis extension to a water stimulus which follows a sucrose stimulus, compared to that given to a water stimulus preceding sucrose (Dethier, Solomon and Turner, 1965; Vargo and Hirsch, 1982). In the blow fly, Phormia regina, CES is positively correlated with classical conditioning of the proboscis extension reflex (Nelson, 1971; McGuire and Hirsch, 1977; Tully, Zawistowski and Hirsch, 1982; McGuire, 1983). CES expression in Phormia is also correlated with one major gene locus (Tully and Hirsch, 1982a,b). CES has been demonstrated in D. melanogaster (Vargo and Hirsch, 1982). Subsequent work has produced Drosophila lines selected for high and low expression of CES (Vargo and Hirsch, 1985) and, as in Phormia, has shown a positive correlation between CES and classical conditioning of the proboscis extension reflex (Holliday, Vargo and Hirsch, 1983). The bio- 
metrical and chromosome analyses described below are the first attempt to identify the genetic correlates of CES in Drosophila.

\section{BEHAVIOURAL TESTING}

On every trial of the CES test, each animal received a 5 second presentation of distilled water applied to the tarsi, followed immediately by a 5 second presentation of $0.25 \mathrm{M}$ sucrose to the tarsi and labellum. Sucrose was followed by a 45 second interstimulus interval (ISI) during which no stimuli were administered. Following the ISI was a 5 second presentation of distilled water (posttest), again applied to the tarsi. The solutions were presented on filter paper strips to be described below. All three strips (pretest, sucrose, and posttest) were at a distance from the flies permitting labellar contact with them. Labellar contact with the strip was required in order for a response to sucrose stimulation to be recorded, though not for a response to water pretest and posttest stimulations. To control for thirst, animals were permitted to imbibe the solutions.

The CES test presented eight trials at a 6 minute intertrial interval (ITI). Proboscis extension (Position 3 or higher on the Dethier et al. scale, 1965) was scored all or none, resulting in scores for pretest, sucrose and posttest ranging 0-8, with posttest being the measure of CES in an animal. All animals were approximately 48 hour old at testing and food-deprived for 36 hours, by placing them in a vial containing water-soaked cotton. Before each test, animals were mounted in micropipette tips (Vargo, Holliday and Hirsch, 1983) and given water $a d$ lib for 15 minute.

Animals were tested on the apparatus described in Vargo, Holliday and Hirsch (1983). The solutions were presented on Whatman No. 3 filter paper strips, placed obliquely on the surface of a vertically positioned kymograph drum. The animals, positioned vertically along the side of the kymograph, were stimulated sequentially when, as the drum rotated and the strips approached each animal, a fly extended its tarsi and walked over the strips. With this apparatus, in one session 18 flies were tested and observed through a stereomicroscope $(9 \times$ magnification).

Animals were discarded from analysis for obtaining a sucrose score below 6 or for failure to participate (i.e., not walking over any of the stimulus strips: pretest, sucrose, or posttest) on three or more trials.
BIOMETRICAL ANALYSIS

\section{Methods}

Drosophila melanogaster from the 12th and 18th generations of lines selected for high (HE) and low (LE) CES, respectively, were used (Vargo and Hirsch, 1985) for a biometrical analysis. All stocks were kept on a $16 / 8$ hour L/D cycle at $25^{\circ} \mathrm{C}$ and 50 per cent $\mathrm{RH}$ and maintained on Instant Drosophila Medium (Formula 4-24, Carolina Biological Supply Co., Burlington, NC).

The crosses in the biometrical analysis are described by Mather and Jinks (1971, p. 287) and summarised in table 1 . The parameters are the midparent $(m)$, additive effects $([d])$, dominance effects $([h])$, interaction effects $([i],[j],[l])$, maternal effects $([\mathrm{dm}],[\mathrm{hm}]), X$-linked effects $([d x],[h x],[i x],[j x],[l x])$, and $Y$-linked effects $([d y])$. They were estimated with a step-wise multiple regression using the matrices of indicator variables as described in Mather and Jinks, (1971).

Flies, used to breed the several generations of the biometrical analysis, were unselected samples from recently ecloded, untested flies. Then, each generation was tested for CES as it ecloded. Lack of randomisation in testing the several generations should not be a serious disadvantage because all flies must meet specified criteria to be included in the analysis.

\section{Results}

Mean posttest scores for males and females from each generation are shown in table 2 . With generations and sex as factors in an analysis of variance, a significant sex effect $\left(F_{1,666}=63.761, M S E=\right.$

Table 1 The matings for the different generations produced in the biometrical analysis

\begin{tabular}{lll}
\hline Generation & $\begin{array}{l}\text { Female } \\
\text { parent }\end{array}$ & $\begin{array}{l}\text { Male } \\
\text { parent }\end{array}$ \\
\hline $\mathrm{P}_{1}$ & $\mathrm{HE}$ & $\mathrm{HE}$ \\
$\mathrm{P}_{2}$ & $\mathrm{LE}$ & $\mathrm{LE}$ \\
$\mathrm{F}_{1}$ & $\mathrm{P}_{1}$ & $\mathrm{P}_{2}$ \\
$\mathrm{~F}_{1}^{\prime}$ & $\mathrm{P}_{2}$ & $\mathrm{P}_{1}$ \\
$\mathrm{~F}_{2}$ & $\mathrm{~F}_{1}$ & $\mathrm{~F}_{1}$ \\
$\mathrm{~F}_{2}^{\prime}$ & $\mathrm{F}_{1}^{\prime}$ & $\mathrm{F}_{1}^{\prime}$ \\
$\mathrm{B}_{1}^{\prime}$ & $\mathrm{P}_{1}$ & $\mathrm{~F}_{1}$ \\
$\mathrm{~B}_{1}^{\prime \prime}$ & $\mathrm{P}_{1}$ & $\mathrm{~F}_{1}$ \\
$\mathrm{~B}_{1}^{\prime \prime \prime}$ & $\mathrm{F}_{1}$ & $\mathrm{P}_{1}$ \\
$\mathrm{~B}_{1}^{\prime \prime \prime}$ & $\mathrm{F}_{1}^{\prime}$ & $\mathrm{P}_{1}^{\prime}$ \\
$\mathrm{B}_{2}^{\prime}$ & $\mathrm{P}_{2}$ & $\mathrm{~F}_{1}$ \\
$\mathrm{~B}_{2}^{\prime \prime}$ & $\mathrm{P}_{2}$ & $\mathrm{~F}_{1}^{\prime}$ \\
$\mathrm{B}_{2}^{\prime \prime \prime}$ & $\mathrm{F}_{1}$ & $\mathrm{P}_{2}$ \\
$\mathrm{~B}_{2}^{\prime \prime \prime}$ & $\mathrm{F}_{1}^{\prime}$ & $\mathrm{P}_{2}$ \\
\hline
\end{tabular}


Table 2 Posttest scores for males and females from the biometrical analysis. Scores represent means + SE

\begin{tabular}{lllll}
\hline Generation & Males & $n$ & Females & $n$ \\
\hline $\mathbf{P}_{1}$ & $7 \cdot 3 \pm 0 \cdot 38$ & 11 & $6 \cdot 5 \pm 0 \cdot 36$ & 17 \\
$\mathbf{P}_{2}$ & $0 \cdot 9 \pm 0 \cdot 26$ & 16 & $1 \cdot 2 \pm 0 \cdot 59$ & 14 \\
$\mathrm{~F}_{1}$ & $3 \cdot 8 \pm 0 \cdot 50$ & 23 & $1 \cdot 5 \pm 0 \cdot 34$ & 27 \\
$\mathrm{~F}_{1}^{\prime}$ & $2 \cdot 7 \pm 0 \cdot 43$ & 27 & $0 \cdot 5 \pm 0 \cdot 17$ & 26 \\
$\mathrm{~F}_{2}$ & $5 \cdot 3 \pm 0 \cdot 40$ & 31 & $3 \cdot 2 \pm 0 \cdot 44$ & 34 \\
$\mathrm{~F}_{2}^{\prime}$ & $4 \cdot 2 \pm 0 \cdot 50$ & 29 & $3 \cdot 2 \pm 0 \cdot 46$ & 33 \\
$\mathbf{B}_{1}^{\prime}$ & $6 \cdot 5 \pm 0 \cdot 24$ & 26 & $4 \cdot 8 \pm 0 \cdot 48$ & 25 \\
$\mathbf{B}_{1}^{\prime \prime}$ & $5 \cdot 2 \pm 0 \cdot 61$ & 25 & $3 \cdot 4 \pm 0 \cdot 51$ & 27 \\
$\mathbf{B}_{1}^{\prime \prime \prime}$ & $4 \cdot 9 \pm 0 \cdot 69$ & 20 & $3 \cdot 6 \pm 0 \cdot 54$ & 27 \\
$\mathbf{B}_{1}^{\prime \prime \prime}$ & $5 \cdot 3 \pm 0 \cdot 42$ & 24 & $3 \cdot 7 \pm 0 \cdot 67$ & 26 \\
$\mathbf{B}_{2}^{\prime}$ & $2 \cdot 8 \pm 0 \cdot 46$ & 27 & $1 \cdot 0 \pm 0 \cdot 30$ & 26 \\
$\mathbf{B}_{2}^{\prime \prime}$ & $1 \cdot 6 \pm 0 \cdot 42$ & 26 & $1 \cdot 8 \pm 0 \cdot 46$ & 27 \\
$\mathbf{B}_{2}^{\prime \prime}$ & $2 \cdot 3 \pm 0 \cdot 44$ & 26 & $1 \cdot 3 \pm 0 \cdot 43$ & 23 \\
$\mathbf{B}_{2}^{\prime \prime \prime}$ & $3 \cdot 6 \pm 1 \cdot 60$ & 26 & $1 \cdot 5 \pm 0 \cdot 39$ & 27 \\
\hline
\end{tabular}

5.498, $P=0.001)$ was found. Therefore, all analyses were performed on the sexes separately.

For the two models generated (table 3), both contain significant midparent, additive, dominance and epistatic components. The male model also contains a significant $Y$ chromosome effect and the female model indicates that maternal and $\mathrm{X}$-linked effects are present.

\section{CHROMOSOME ANALYSIS}

\section{Methods}

From HE and LE separately, six experimental groups $(1,2,3,4,5$ and 6$)$ were produced containing one or two pairs of either HE or LE chromosomes (X, II, or III) on a control-line genetic background-1, X; 2, II; 3, III; 4, X and II; 5, X and III; 6 , II and III. The fourth chromosome was not studied.

Table 3 Best fit models derived from the biometrical analysis for males and females. Values represent parameter estimates. All estimates are significantly different from zero ( $t$-test; $P<0.05$ ). Chi-square values with their corresponding $P$ values for each model are also shown

\begin{tabular}{lcc}
\hline Parameter & Male & Female \\
\hline$[m]$ & $5 \cdot 96$ & $5 \cdot 79$ \\
{$[d]$} & $3 \cdot 74$ & $2 \cdot 06$ \\
{$[h]$} & $-2 \cdot 85$ & $-3 \cdot 85$ \\
{$[i]$} & $-1 \cdot 91$ & $-1 \cdot 69$ \\
{$[d m]$} & - & 0.48 \\
{$[h x]$} & - & -0.92 \\
{$[d y]$} & -0.57 & - \\
$X^{2}$ & $4 \cdot 214$ & $7 \cdot 149$ \\
$P$ & 0.897 & 0.521 \\
\hline
\end{tabular}

Flies from the 20th, 26th, and 11th generations of $\mathrm{HE}, \mathrm{LE}$, and the control line, respectively, were used (Vargo and Hirsch, 1985). The multiple inversion balancer stock used to the produce the experimental groups was: Basc; $\operatorname{In}(2 \mathrm{LR}) \mathrm{bw}^{\mathrm{VI}}, \mathrm{ds}^{33 \mathrm{k}} \mathrm{dp}$ b bw ${ }^{\mathrm{V} 1} / \mathrm{SM} 1$; In(3R)C, Sb/TM2; obtained from the University of Utah (Drosophila Information Service, 1982, Ref. No. 11180). The different chromosome types were produced using the method of Robertson (1954).

\section{Results}

As before, a significant sex effect was present (Anova, $\quad \mathrm{F}_{1,298}=28 \cdot 265, \quad \mathrm{MSE}=4.910, \quad P=$ 0.001 ); therefore, male and female results were analysed separately. Scores from each group in each line for males and females are presented in table 4. All groups were tested from one generation except Group 3 in $\mathrm{HE}$, which was tested in two successive generations. The large discrepancies in sample sizes between males and females and among groups are a result of differences in viability among the groups. In fact, three attempts to produce Group 4 (Chromosomes X and II) from LE were unsuccessful. Despite these differences in viability, the differences reported between groups are primarily differences in CES and not due to the health of the flies because, for a fly's score to be included in the analysis, it has to meet specified criteria (food deprivation, walking ability, and sucrose response). As a result, those flies that do qualify are quite vigorous.

An analysis of variance with group, line, and sex as factors revealed that all three factors and

Table 4 Mean posttest scores for males and females from each group in the chromosome analysis of each line. Mean posttest scores are shown with their standard errors and with sample size indicated below. The selected line chromosome content of each group is: $1,(\mathrm{X}) ; 2$, (II); 3, (III); 4, (X and II); 5, (X and III); 6, (II and III)

\begin{tabular}{|c|c|c|c|c|}
\hline Group & Male & $\begin{array}{l}\mathrm{HE} \\
\text { Female }\end{array}$ & Male & $\begin{array}{l}\text { LE } \\
\text { Female }\end{array}$ \\
\hline 1 & $\begin{array}{l}4.9 \pm 0.67 \\
14\end{array}$ & $\begin{array}{l}4 \cdot 7 \pm 0 \cdot 63 \\
17\end{array}$ & $\begin{array}{l}6 \cdot 5 \pm 0 \cdot 47 \\
12\end{array}$ & $\begin{array}{l}4.9 \pm 0.54 \\
13\end{array}$ \\
\hline 2 & $\begin{array}{l}7 \cdot 6 \pm 0 \cdot 22 \\
10\end{array}$ & $\begin{array}{l}6 \cdot 2 \pm 0 \cdot 43 \\
21\end{array}$ & $\begin{array}{l}4 \cdot 9 \pm 0 \cdot 46 \\
10\end{array}$ & $\begin{array}{l}3 \cdot 0 \pm 0 \cdot 54 \\
12\end{array}$ \\
\hline 3 & $\begin{array}{l}4.9 \pm 0.88 \\
7\end{array}$ & $\begin{array}{l}2 \cdot 4 \pm 0.66 \\
14\end{array}$ & $\begin{array}{l}5 \cdot 4 \pm 0 \cdot 50 \\
15\end{array}$ & $\begin{array}{l}3 \cdot 1 \pm 0 \cdot 65 \\
17\end{array}$ \\
\hline 4 & $\begin{array}{l}4 \cdot 6 \pm 1 \cdot 36 \\
5\end{array}$ & $\begin{array}{l}3 \cdot 6 \pm 0 \cdot 71 \\
14\end{array}$ & - & - \\
\hline 5 & $\begin{array}{l}6 \cdot 9 \pm 0 \cdot 36 \\
17\end{array}$ & $\begin{array}{l}5 \cdot 2 \pm 0 \cdot 64 \\
15\end{array}$ & $\begin{array}{l}5 \cdot 0 \pm 0 \cdot 58 \\
23\end{array}$ & $\begin{array}{l}4 \cdot 2 \pm 0 \cdot 62 \\
21\end{array}$ \\
\hline 6 & $\begin{array}{l}7 \cdot 5 \pm 0 \cdot 18 \\
13\end{array}$ & $\begin{array}{l}5 \cdot 7 \pm 0 \cdot 57 \\
20\end{array}$ & $\begin{array}{l}1 \cdot 7 \pm 0.45 \\
18\end{array}$ & $\begin{array}{l}0 \cdot 6 \pm 0 \cdot 26 \\
12\end{array}$ \\
\hline
\end{tabular}


the group by line interaction were significant. Focusing on Groups 1,2, and 3 (Chromosomes $\mathrm{X}, \mathrm{II}$, and III, respectively) for $\mathrm{HE}$ males and females, it can be seen that Group 2 (Chromosome II) gave the highest CES score, suggesting that residing on Chromosome II are loci of major effect correlated with CES expression. If so, we would expect Groups 4 (X and II) and 6 (II and III) likewise to demonstrate high CES. For both males and females, Group 6 showed fairly high CES scores; however, the low scores of Group 4 and the unexpectedly high scores of Group 5 (X and III) can not be explained unless a model involving epistasis among all three chromosomes is involved. With a posteriori comparisons (Tukey, $P=0.05$ ), the highest scoring homogeneous subset of groups for HE males contained Groups 2 (II), 6 (II and III), and 5 ( $\mathrm{X}$ and III), while for females the highest homogeneous subset contained Groups 2 (II), 6 (II and III), 5 (X and III), and 1 (X).

With regard to the LE groups, a posteriori comparisons revealed that the lowest homogeneous subset for males contained only Group 6 (II and III), whereas the lowest homogeneous subset for females contained two groups; 6 (II and III) and 2 (II). The large difference, however, between the scores of Group 6 (Mean $=0.6$ ) and Group 2 $($ Mean $=3.0)$ for the low females, combined with the low score of Group 6 for the males, indicates that an interaction between Chromosomes II and III is primarily responsible for low CES in this line.

\section{DISCUSSION}

The above studies have identified some of the genetic correlates of CES in Drosophila melanogaster. First, it was determined from the biometrical analysis that low expression is partially dominant to high expression. This interpretation is made from the negative dominance component in the model and reflected in the reciprocal $F_{1}$ scores for males and females (table 2) which are closer to the scores of LE than to those of HE. This dominance component, however, is unstable across generations of selection. During the course of this work, four $F_{1}$ populations were produced, at the 16th, 18th, 22nd, and 26th generations of selection in the low line. But the additional $F_{1}$ populations were not part of complete biometrical analyses. Nevertheless it can be seen (fig. 1) that the $F_{1}$ scores increased across generations, even though no significant change occurred in the scores of the HE and LE lines.
Second, the biometrical analysis revealed that a significant Y-linked effect is present. Of the 14 different generations, the generations $B_{1}^{\prime}$ and $B_{1}^{\prime \prime}$ are identical with regard to $\mathrm{X}$ chromosome, autosomal, and cytoplasmic complements. The only difference between $B_{1}^{\prime}$ and $B_{1}^{\prime \prime}$ is in regard to their $Y$ chromosome content. $B_{1}^{\prime}$ has an LE Y chromosome whereas $B_{1}^{\prime \prime}$ has an HE Y chromosome. The same can be said for the $B_{2}^{\prime}$ and $B_{2}^{\prime \prime}$ generations which are identical except for the presence of the LE or HE Y chromosome, respectively. From these two comparisons, the generations with the low $\mathrm{Y}$ chromosome ( $\mathrm{B}_{1}^{\prime}$ and $\mathrm{B}_{2}^{\prime}$ ) have higher scores then their complementary generations with the high $\mathrm{Y}$ chromosome ( $B_{1}^{\prime \prime}$ and $B_{2}^{\prime \prime}$ ). This effect is represented in the biometrical analysis by a negative estimate of the parameter $[d y]$. In the chromosome analysis, because the crosses used involved mating $\mathrm{HE}$ and LE females to balanced males, no information is obtained about the $\mathrm{Y}$ chromosome.

Third, the biometrical analysis revealed a signficant cytoplasmic effect, which is reflected in the mean scores of the reciprocal $F_{1}$ females. The $F_{1}$ generation, which contains a high cytoplasmic complement, has a mean posttest score higher than that of $F_{1}^{\prime}$ which contains the low cytoplasmic complement.

Lastly, the biometrical analysis indicated that some X-linked effects for CES exist. This conclusion is based on the low values for the $\mathrm{X}$-linked parameter estimates in the analysis and the inconsistent results from $\mathrm{HE}$ in the chromosome analysis. The exact nature of the X-linked effect can not be explained at this time.

The chromosome analysis revealed that chromosome II has a major effect for high CES expression. It also revealed that an interaction involving chromosomes X and III is likewise correlated with high CES. The results from LE indicated that an epistasis involving chromosomes II and III is correlated with low CES expression; which was also suggested by the significant interaction effect in the biometrical analysis.

This combination of biometrical and chromosome analyses has proven to be useful for finding the genetic correlates of a behaviour-trait. Biometrical analysis acts as a first approximation in determining the experiments required to find the genetic correlates of a behaviour. In the present study, for instance, the biometrical analysis suggested a strong epistatic effect, which could be further explored by the chromosome analysis. The results of the chromosome analysis not only corroborated the effect but also identified those chromosomes involved. More importantly, the bio- 


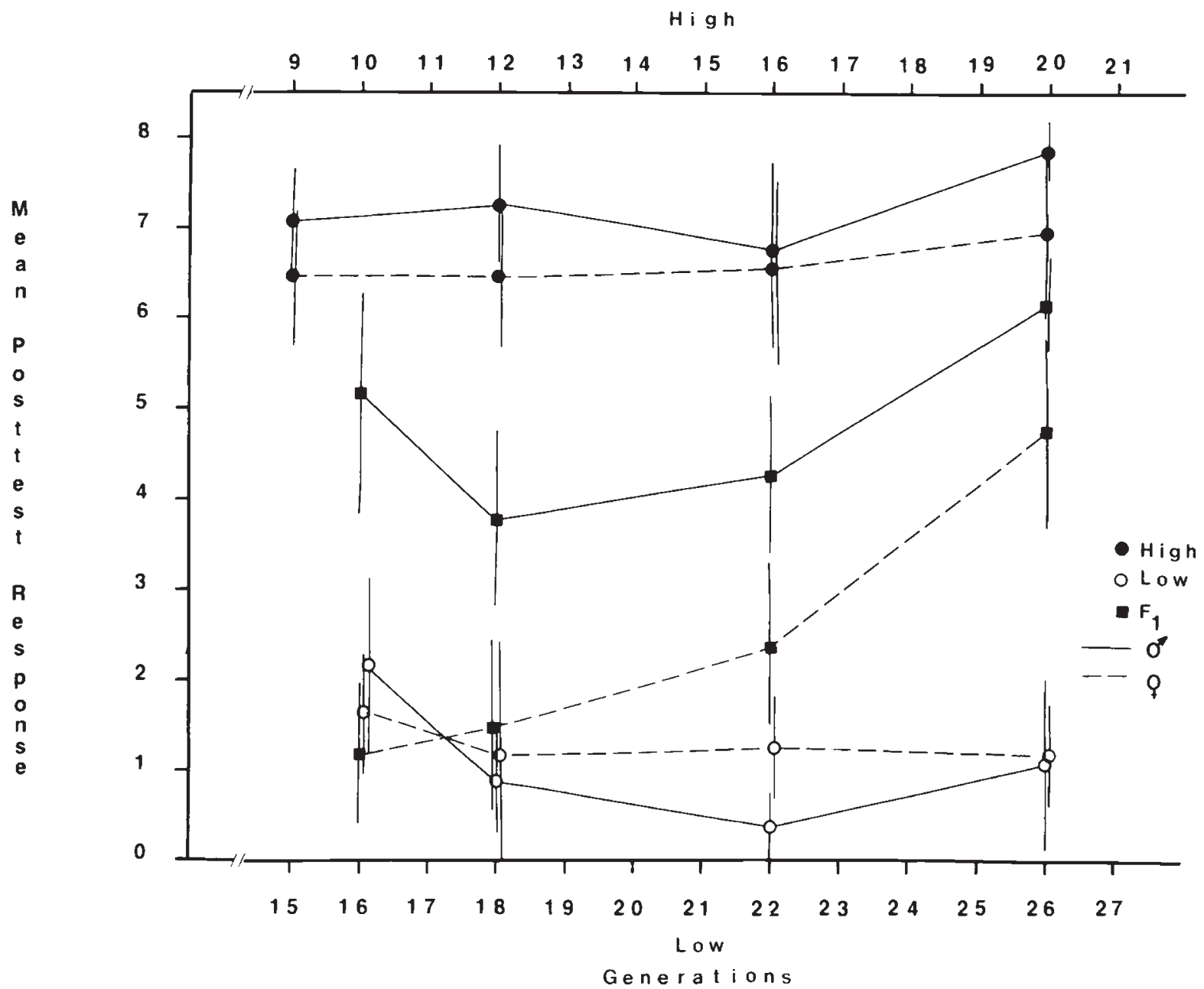

Figure 1 Male and female mean post-test scores for HE, LE, and resulting $F_{1}$ progeny from the biometrical analysis as well as the three additional hybrid crosses. The abcissa represents LE, whereas HE generations are shown atop the figure. HE was started seven generations later than LE.

metrical analysis is sensitive enough to detect minor differences which would not be detected merely by comparing the means of various generations. For example, comparisons of the female means from reciprocal $F_{2}$ generations result in no significant difference, suggesting no sex-linked effect. The chromosome analysis indicated that some X-linked loci were involved, though it is unsure what the exact nature of the $\mathrm{X}$ effect is. The biometrical analysis identified the sex-linked effect before it was corroborated by the chromosome analysis.

Finally, the complexity of the genetic system correlated with CES expression in these Drosophila lines is markedly different from the relatively simple system found in Phormia. Tully and Hirsch (1982b) showed in Phormia that one major locus, with the heterozygote showing semidominance, is correlated with CES expression, but that no effects on CES expression were found for X-linked, Ylinked, and cytoplasmic components. Whether the vast difference in complexity between the two species is a result due to long laboratory maintenance of the stocks or resembles differences present in natural populations is not known at present.

Acknowledgements This work was supported by grants from the National Science Foundation (BNS-83-00353), the National Institute of Mental Health for training in research on Institutional Racism (MH15173), and the National Institutes of Health (NIH RR 7030) for Biomedical Research Support. 


\section{REFERENCES}

DETHIER, V. G., SOLOMON, R. L. AND TURNER, L. H. 1965 Sensory input and central excitation in the blowfly. Journal of Comparative and Physiological Psychology, 60, 303-313.

HIRSCH, J. 1959. Studies in experimental behavior genetics. II. Individual differences in geotaxis as a function of chromosome variations in synthesized Drosophila populations. Journal of Comparative and Physiological Psychology, 52, 304-308.

HIRSCH, J. AND ERLENMEYER-KIMLING, L. 1962. Studies in experimental behavior genetics: IV. Chromosome analyses for geotaxis. Journal of Comparative and Physiological Psychology, 55, 732-739.

HIRSCH, J. AND KSANDER, G. 1969. Studies in experimental behavior genetics: V. Negative geotaxis and further chromosome analyses in Drosophila melanogaster. Journal of Comparative and Physiological Psychology, 67, 118-122.

HOllidAY, M., VARGO, M., AND HiRSCH, J. 1983, April. A hybrid-correlational analysis using classical conditioning and central excitatory state (CES) of the fruit fly, (Drosophila melanogaster). Paper presented at the Midwest Regional Animal Behavior Meeting, University of Missouri-St. Louis.

MATHER, K. AND HARRISON, B. J. 1949. The manifold effects of selection. Heredity, 3, 131-162.

MATHER, K. AND JINKS, J. L. 1971. Biometrical Genetics (2nd edition), Chapman and Hall, London.

MCGUIRE, T. R. 1983. Further evidence for a relationship between central excitatory state and classical conditioning in the blow fly, Phormia regina. Behavior Genetics, 13, 509-515.

MCGUIRE, T. R. AND HIRSCH, J. 1977. Behavior-genetic analysis of Phormia regina: Conditioning, reliable individual differences, and selection. Proceedings of the National Academy of Sciences of the United States of America, 74, 5193-5197.

NELSON, M. C. 1971. Classical conditioning in the blowfly (Phormia regina): Associative and excitatory factors. Journal of Comparative and Physiological Psychology, 77, 353368.
PYLE, D. 1978. A chromosome substitution analysis of geotactic maze behavior in Drosophila melanogaster. Behavior Genetics, 8, 53-64.

ROBERTSON, F. W. 1954. Studies in quantitative inheritance: V. Chromosome analysis of crosses between selected and unselected lines of different body size in Drosophila melanogaster. Journal of Genetics, 52, 494-520.

TULLY, T. AND HIRSCH, J. 1982. Behavior-genetic analysis of Phormia regina. I. Isolation of pure breeding lines for high and low levels of the central excitatory state (CES) from an unselected population. Behavior Genetics, 12, 395-416. (a).

TULLY, T. AND HIRSCH, J. 1982. Behavior-genetic analysis of Phormia regina II. Detection of a single, major-gene effect from behavioural variation for central excitatory state (CES) using replicate hybrid crosses. Animal Behaviour, 30, 1193-1202. (b).

TULLY, T., ZAWISTOWSKI, S. AND HIRSCH, J. 1982. Behaviorgenetic analysis of Phormia regina: III. A phenotypic correlation between the central excitatory state (CES) and conditioning remains in replicated $F_{2}$ generations of hybrid crosses. Behavior Genetics, 12, 181-191.

VARGO, M. 1985. Behavior-genetic analysis of Drosophila melanogaster using the central excitatory state (Doctoral dissertation, University of Illinois at Urbana-Champaign, 1984). Dissertation Abstracts International, 45(11), 3655-B.

VARGO, M. AND HIRSCH, J. 1982. Central excitation in the fruit fly (Drosophila melanogaster). Journal of Comparative and Physiological Psychology, 96, 452-459.

VARGO, M. AND HIRSCH, J. 1985. Selection for central excitation in Drosophila melanogaster. Journal of Comparative Psychology, 99, 81-86.

VARGO, M., HOllidAY, M. AND HiRSCH, J. 1983. Automatic stimulus presentation for the proboscis extension reflex in Diptera. Behavior Research Methods \& Instrumentation, 15 , $1-4$. 